DOI: $10.3901 / J M E .2020 .10 .144$

\title{
空气弹簧弹射冲击性能影响因素研究
}

\author{
李玉如 ${ }^{1,2}$ 杨 冰 ${ }^{1}$ 谢君科 $^{3}$ 肖守讷 ${ }^{1}$ 阳光武 ${ }^{1}$ 朱 涛 $^{1}$ \\ 肖世德 ${ }^{2}$ 柳忠涁 ${ }^{3}$
}

(1. 西南交通大学牵引动力国家重点实验室 成都 610031;

2. 西南交通大学机械工程学院 成都 610031;

3. 四川轻化工大学机械工程学院 宜宾 644000)

\begin{abstract}
摘要: 基于空气弹簧的非线性特性, 建立一种流固耦合弹射模型, 并通过弹射碰撞试验系统验证模型的准确性。基于该模 型, 研究初始压强、压缩位移对空气弹簧弹射冲击性能的影响, 再现了空气弹簧在弹射过程中的往复振荡现象, 同时表明 通过调整初始压强和压缩位移能够显著提高弹射冲击性能。进一步, 通过改变模型加强筋参数, 分析帘线角、帘线层、帘 线间距、帘线直径和距中性面距离等参数对空气弹簧弹射冲击性能的影响。结果显示, 帘线参数对弹射冲击性能的影响有 限, 但帘线层数的增加可显著提高空气弹簧稳定性。分析结果有助于拓宽空气弹簧的工程应用范围, 促进相关碰撞测试的 开展和车辆被动安全性能提升。
\end{abstract}

关键词: 空气弹簧; 弹射冲击性能; 往复振荡; 帘线参数; 被动安全

中图分类号: U270

\section{Study of Influence Factors on Ejection Impact Performance of Air Spring}

\author{
LI Yuru $^{1,2}$ YANG Bing $^{1} \quad$ XIE Junke $^{3} \quad$ XIAO Shoune $^{1} \quad$ YANG Guangwu $^{1}$ ZHU Tao ${ }^{1}$ \\ XIAO Shide $^{2}$ LIU Zhongbin ${ }^{3}$
}

(1. State Key Laboratory of Traction Power, Southwest Jiaotong University, Chengdu 610031;

2. School of Mechanical Engineering, Southwest Jiaotong University, Chengdu 610031;

3. School of Mechanical Engineering, Sichuan University of Science \& Engineering, Yibin 644000)

\begin{abstract}
Based on the nonlinear characteristics of air spring, a fluid-structure coupled ejection model is established, and the accuracy of the model is verified by ejection impact test system. Based on this model, the influence of initial pressure and compression displacement on the ejection impact performance of air spring is studied, and the phenomenon of reciprocating oscillation of air spring in the ejection process is reproduced. It is shown that the ejection impact performance of air spring can be significantly improved by adjusting the initial pressure and the compression displacement. Furthermore, the influence of cord angle, cord layer, cord spacing, cord diameter and distance from the middle surface on the air spring ejection performance is analyzed by changing the parameters of the rebar model. The results show that the influence of cord parameters on ejection impact performance is limited, but the increase of cord layers can improve the stability of air spring effectively. The analysis results are helpful to broaden the engineering application of air spring, promote the development of relevant crash test and improve the passive safety performance of vehicles.
\end{abstract}

Key words: air spring; ejection impact performance; reciprocating oscillation; cord parameters; passive safety

\section{0 前言}

随着高速铁路持续发展, 车辆运行速度不断提

\footnotetext{
* 国家重点研发计划课题(2016YFB1200403-A03)、四川省科技计划 (2019YJ0216)、牵引动力国家重点实验室自主课题(2017TPL_Z01)和 四川轻化工大学人才引进(2016RCL23)资助项目。20190715 收到初 稿, 20200305 收到修改稿
}

高, 列车被动安全问题越来越受到人们的重视, 其中, 吸能部件和缩比车辆的碰撞试验是研究热 点之一。对于吸能部件, 需要通过试验获得力-位 移曲线来评估吸能装置的吸能性能是否满足设计 要求; 对于缩比车辆, 需要获得结构变形、速度、 加速度、界面力等参数, 为车辆耐撞性设计提供 参考依据。为开展相关研究, 需要能在短时间内 提供较高速度的动力弹射装置, 并应具有弹射速 
度范围广、反应时间短、噪声小、安全性高等特 点, 当前这类设备较为紧缺。空气弹簧因其具有 反应迅速、耐高压、变刚度以及隔声性能好等特 点, 是弹射驱动装置的重要可选件 ${ }^{[1]}$ 。研究压强、 压缩位移和帘线参数等关键因素对空气弹簧弹射 冲击性能的影响, 有助于被动安全测试装备动力 源选型和优化, 促进相关测试开展和车辆被动安 全性能提升。

空气弹簧作为铁道车辆常用部件, 在连接转向 架和车体的二系悬挂系统中广为应用, 围绕其对车 辆动力学性能影响的研究也相对成熟。吴兴文等 ${ }^{[2]}$ 研究了三种空气弹簧模型对机车车辆直线运行平稳 性和曲线通过安全性的影响。RYABOY ${ }^{[3]}$ 研究了 空气弹簧的垂向动态特性, 并分析由多个空气弹 簧构成的隔振系统稳定性。陈燎等 ${ }^{[4]}$ 提出了一种 空气弹簧动态特性拟合和悬架模型非线性仿真的 方法。FACCHINETTI 等 ${ }^{[5]}$ 定义和比较了二级空气 弹簧悬架的两种建模方法, 并评估它们对轨道车 辆动力学多体仿真精度的影响。CHANG 等 ${ }^{[6]}$ 建立 了空气弹簧的动力学模型, 并将其集成到整车多 体动力学模型中, 以研究空气悬架行为和车辆动 力学特性。

此外, 空气弹簧等效模型构建方法的研究也是 当前热点之一。ZENG 等 ${ }^{[7]}$ 通过试验获得一种高阶 非线性回归方法, 实现了双气室空气弹簧刚度和阻尼 的快速计算。LI 等 ${ }^{[8]}$ 根据所提出的公式研究了几何参 数对空气弹簧垂直刚度的影响。BHATTACHARYYA 等 ${ }^{[9]}$ 研究空气弹簧刚度线性化对阻尼器性能的影 响, 并给出不同设计参数下阻尼器的频率变化。李 䒾等 ${ }^{[10]}$ 基于热力学和流体力学理论, 建立空气弹簧 物理模型, 研究了主要因素对空气弹簧性能的影 响。顾太平等 ${ }^{[11]}$ 提出波纹管式空气弹簧平衡性能的 计算方法, 并研究窝线角度对平衡性能的影响。ZHU 等 ${ }^{[12]}$ 提出了空气弹簧动力学模型, 通过试验得到了 模型参数, 并通过对几种空气弹簧模型的对比验证 模型的正确性。QUAGLIA 等 ${ }^{[13]}$ 推导出囊式回转体 空气弹簧刚度的表达式, 并应用图解法详细分析 空气弹簧的有效面积变化情况。徐国敏等 ${ }^{[14]}$ 提出 囊体耐压强度简化计算公式, 并验证了理论模型 的正确性。

近年来, 德国 SCHENCK、美国 HYGE、MTS、 IST SCHENCK、三菱重工、SEATTLYSAFTY、西 雅图公司、中南大学和中车青岛四方机车车辆股份 有限公司等 ${ }^{[15]}$ 相继进行液压驱动、气缸驱动等碰撞 试验设备的开发, 但普遍存在冲击响应时间久、音
爆、速度控制精度差等缺点, 空气弹簧作为一种新 型弹射冲击驱动部件能够弥补上述缺点, 然而现有 针对其冲击性能的研究还较为有限。李建阳等 ${ }^{[16]}$ 对 2 种自充气式气囊进行室内投放冲击试验, 得到了 气囊最大压强与着陆初速度和载荷质量之间的关 系。袁春元等 ${ }^{[17]}$ 提出一种空气弹簧力学特性预估方 法, 并通过试验验证了其方法的可行性。MELO 等 ${ }^{[18]}$ 给出汽车悬架用波纹管式空气弹簧变形的数值解, 并模拟气动悬架波纹管在动载荷作用下的行为。吴 善跃等 ${ }^{[19]}$ 通过落锤冲击试验得出空气弹簧在快速 变形影响下的冲击载荷特性明显硬于静载荷特性。 柳忠涁等 ${ }^{[20]}$ 提出了基于压缩气体研究多节气囊的 受力弹射过程和行为变化。LI 等 ${ }^{[21]}$ 通过仿真研究了 气体质量流和壁厚对柔性气囊弹射性能的影响, 得 出增加质量流能够提高弹射速度, 而壁厚的改变对 弹射性能的影响较小的结论。

综上可知, 由于空气弹簧弹射过程的强非线性 特性, 现有研究多通过试验进行, 传统理论方法对 其弹射特性的计算效率较低, 给空气弹簧的设计和 优化带来困难, 在一定程度上限制其在弹射领域的 应用。本文通过建立经试验验证的气固耦合空气弹 簧仿真模型, 对其弹射冲击性能进行研究, 并从初 始气体压强、压缩位移和窝线参数设置等方面为冲 击性能的提升提出改进建议。

\section{1 空气弹簧模型}

\section{1 空气弹簧弹射模型}

如图 1 所示, 空气弹簧弹射模型由三曲囊单元 $(\mathrm{N} 1 、 \mathrm{~N} 2 、 \mathrm{~N} 3)$ 、固定刚性墙和移动体组成。曲囊单 元之间相互连通, 形成相对封闭的气囊腔室。其中 单元 N1 包含进气孔并与固定刚性墙相连; 单元 N3 与移动体接触, 通过对空气弹簧通入高压气体, 推 动移动体沿 $X$ 方向加速弹射。

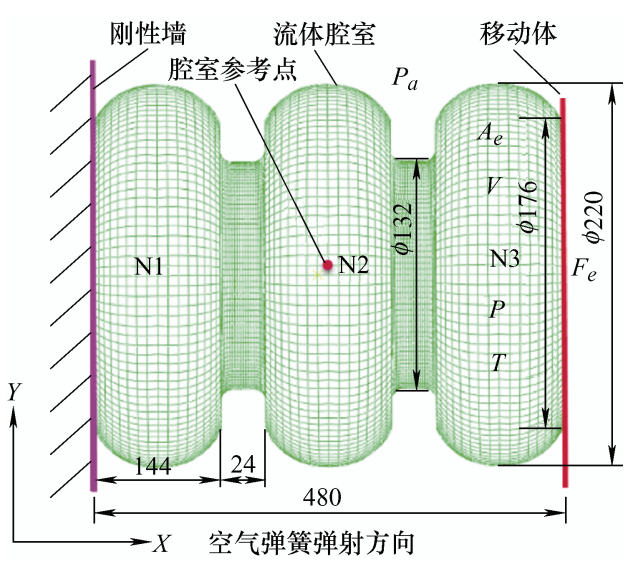

图 1 空气弹簧弹射系统及气固耦合有限元模型 
图 1 中, $P$ 和 $P_{a}$ 分别表示腔室的绝对压力和大 气压力; $T$ 为腔室温度; $F_{e}$ 为沿 $X$ 方向的轴向弹射 力; $V$ 为空气弹簧腔室体积。

空气弹簧在三个方向上允许偏转和力的传递 ${ }^{[22]}$, 对弹射冲击性能起决定作用的是轴向弹射力

$$
F_{e}=\left(p-p_{a}\right) A_{e}
$$

空气弹簧与移动体的有效接触面积 $A_{e}$ 被定义 为 $X$ 方向上的位移函数

$$
A_{e}=A_{e}(x)
$$

在空气弹簧和外界系统没有气体交换情况下, 依据热力学定律, 由理想气体方程可知

$$
P V^{n}=\text { const }
$$

式中, 等温条件下 $n=1$, 绝热条件下 $n=1.4$, 多变条 件下 $1<n<1.4$ 。

空气弹簧腔室初始压力 $P_{0}$ 和初始体积 $V_{0}$, 压力 为 $P$ 时, 空气弹簧腔室体积 $V$ 为

$$
V=V_{0}\left(\frac{P_{0}}{P}\right)^{\frac{1}{n}}
$$

由式(4)对 $x$ 进行微分可得

$$
\begin{gathered}
V^{n} \frac{\mathrm{d} P}{\mathrm{~d} x}+n P V^{n-1} \frac{\mathrm{d} V}{\mathrm{~d} x}=0 \\
\frac{\mathrm{d} P}{\mathrm{~d} x}=-\frac{n P}{V} \frac{\mathrm{d} V}{\mathrm{~d} x}
\end{gathered}
$$

式(2)进行微分后替换式(6)得到空气弹簧非线 性弹性刚度与力、位移关系如下

$$
K_{e}=\frac{\mathrm{d} F}{\mathrm{~d} x}=-\frac{n P A_{e}}{V} \frac{\mathrm{d} V}{\mathrm{~d} x}+\left(P-P_{a}\right) \frac{\mathrm{d} A_{e}}{\mathrm{~d} x}
$$

\section{2 气压流体模型}

空气弹簧弹射性能不仅取决于几何非线性、材 料非线性和接触非线性 ${ }^{[23]}$, 还取决于腔室压力变 化。通常采用有限元法对结构建立壳单元, 对腔室 采用基于面的流体建模方法实现充气模拟 ${ }^{[24]}$ 。

基于面的流体单元覆盖在流体腔边界上, 每个 腔室均有唯一的腔室参考点和单一自由度指代流体 腔压力, 对于每个腔室需要定义流体密度、体积模 量、膨胀系数等参数。流体单元可用于模拟气动条 件下各种充气腔, 并根据增广虚功原理, 利用理想 气体方程确定密闭气体边界条件, 结构的虚功方程 通过气体边界条件得出 ${ }^{[25-26]}$ 。

流体体积与流体压力、温度和质量构成一种函 数关系。通过流体压力 $P$ 和气体温度 $\theta$ 获得流体腔 体积 $\bar{V}$, 与实际腔室体积 $V$ 相等。在非线性有限元
软件 ABAQUS/STAND 中, 结构的增广虚功表达式 通过如下约束方程来实现 ${ }^{[27]}$

$$
\begin{gathered}
\bar{V}=f(p, \theta, m) \\
V=\bar{V}
\end{gathered}
$$

除此之外, 给出腔室压力引起的虚功贡献

$$
\delta \Pi^{*}=\delta \Pi-p \delta V-\delta p(V-\bar{V})
$$

式中, $\delta \Pi^{*}$ 为增广虚功表达式, $\delta \Pi$ 为无流体腔结 构虚功表达式, 负号意味着腔室体积增加会释放流 体能量, 这是一种混合公式, 其中结构位移和流体 压力是主要变量, 得到增广虚功速率

$$
\begin{array}{r}
\mathrm{d} \delta \Pi^{*}=\mathrm{d} \delta \Pi-p \mathrm{~d} \delta V-\mathrm{d} p \delta V-(\mathrm{d} V-\mathrm{d} \bar{V}) \delta p \\
\mathrm{~d} \delta \Pi^{*}=\mathrm{d} \delta \Pi-p \mathrm{~d} \delta V-\mathrm{d} p \delta V-\mathrm{d} V \delta p+\frac{\mathrm{d} \bar{V}}{\mathrm{~d} p} \mathrm{~d} p \delta p \\
\delta \Pi^{*}=\delta \Pi-p \sum_{e} \delta V^{e}-\delta p\left[\sum_{e} V^{e}-\sum_{e} \bar{V}^{e}\right]
\end{array}
$$

式中, $p \mathrm{~d} \delta V$ 为压力载荷刚度, $\mathrm{d} \bar{V} / \mathrm{d} P$ 为流体的体 积-压力顺应性, 由于腔室所有单元压力均相同, 因 此可将增广虚功表达式写成单个单元表达式之和

$$
\delta \Pi^{*}=\sum_{e}\left[\delta \Pi^{e}-p \delta V^{e}-\delta p\left(V^{e}-\bar{V}^{e}\right)\right]
$$

由于腔室所有单元温度均相同, 因此对于每个 单元的流体体积可通过下式计算

$$
\bar{V}^{e}=\bar{V}\left(p, \theta, m^{e}\right)
$$

式中, $m^{e}$ 为单元质量, 实际腔室体积可通过联立方 程式(7)和(11)得出

$$
V=\sqrt[e]{\bar{V}\left(P, \theta, m^{e}\right)}
$$

上述空气弹簧模型及相关方程为有限元建模与 分析提供了理论支持。

\section{2 空气弹簧弹射过程}

\section{1 空气弹簧弹射物理过程}

空气弹簧弹射过程分为压缩位移、充入气体和 移动体弹射, 其弹射过程如图 2 所示。首先通过移 动体对空气弹簧压缩一定位移并约束全部自由度, 之后对空气弹簧充入一定压力气体, 最后释放移动 体在 $X$ 方向的平动自由度约束, 具有一定压力的空 气弹簧推动移动体加速弹射。

图 2 中, $\mathrm{A} 1$ 为初始状态 $\mathrm{A} 2$ 为压缩状态 $\mathrm{A} 3$ 为弹射完成状态。空气弹簧压缩过程由 $a$ 到 $b$, 弹 射过程由 $\mathrm{b}$ 到 $\mathrm{c}$ 。 


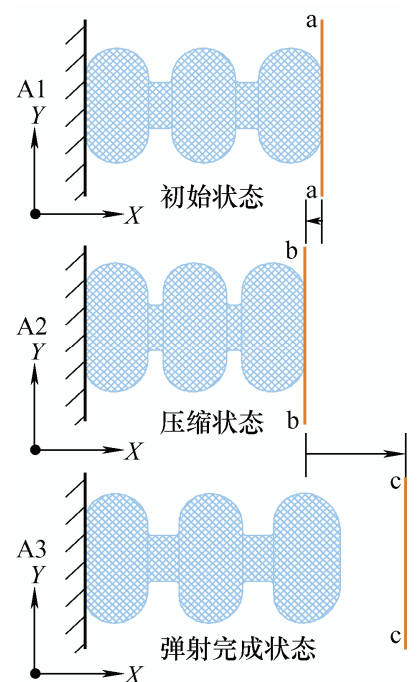

图 2 空气弹簧弹射过程示意图

\section{2 空气弹簧弹射过程有限元模拟}

LS-DYNA 是一种常用的非线性求解软件, 可 以实现对空气弹簧等柔性气囊压缩位移进行仿真计 算 ${ }^{[21]}$, 但无法考虑帘线参数对空气弹簧的影响。因 此, 本文采用 ABAQUS 软件开展仿真分析, 建立 空气弹簧弹射有限元模型(图 1)并设置边界条件和 材料属性。采用 $3 \mathrm{~mm}$ 壳单元 S4R 建立空气弹簧有 限元模型。刚性墙和移动体分别简化为 $240 \mathrm{~mm} \times$ $240 \mathrm{~mm} \times 4 \mathrm{~mm}$ 刚性板, 移动体和刚性墙的质量均 为 $100 \mathrm{~kg}$, 且均采用 $10 \mathrm{~mm}$ 刚性壳单元 R3D4 建 模。移动体与空气弹簧的摩擦因数设为 0.15 , 与地 面的摩擦因数设为 0.1 , 用于模拟试验台车与轨道梁 的摩擦。空气弹簧为橡胶复合材料, 弹射模型基本 材料参数如表 1 所示, 基本流体参数如表 2 所示。

橡胶可视为各项同性材料, 其超弹性力学特性可 用 Mooney-Rivlin 模型 ${ }^{[28]}$ 应变能密度函数 $W$ 来表示

$$
W=C_{10}\left(I_{1}-3\right)+C_{01}\left(I_{2}-3\right)
$$

均为超弹性材料参数。通过拉伸试验获得橡胶材料 参数 $C_{10}=0.8 \mathrm{MPa}, C_{01}=3.2 \mathrm{MPa}$ 。

表 1 空气弹簧弹射模型基本参数

\begin{tabular}{|c|c|c|c|c|c|c|c|}
\hline $\begin{array}{c}\text { 弹射系统 } \\
\text { 部件 }\end{array}$ & $\begin{array}{c}\text { 密度 } \rho / \\
\left(\mathrm{kg} \cdot \mathrm{m}^{-3}\right)\end{array}$ & $\begin{array}{c}\text { 弹性模量 } \\
E / \mathrm{GPa}\end{array}$ & $\begin{array}{c}\text { 泊松 } \\
\text { 比 }\end{array}$ & $\begin{array}{c}\text { 超弹性 } \\
\text { 材料参 } \\
\text { 数 } C_{10} / \\
\mathrm{MPa}\end{array}$ & $\begin{array}{c}\text { 超弹性 } \\
\text { 材料参 } \\
\text { 数 } C_{01} / \\
\mathrm{MPa}\end{array}$ & $\begin{array}{c}\text { 不可压 } \\
\text { 缩常数 } \\
D \\
D\end{array}$ & $\begin{array}{l}\text { 质量 } \\
m / \mathrm{kg}\end{array}$ \\
\hline 移动体 & - & - & - & - & - & - & 100 \\
\hline 固定墙 & - & - & - & - & - & - & 100 \\
\hline 空气弹簧 & 1150 & - & - & 0.8 & 3.2 & 0 & 1.8 \\
\hline 帘线 & 1100 & 2.5 & 0.4 & - & - & - & - \\
\hline
\end{tabular}

表 2 空气弹簧腔室气体参数

\begin{tabular}{ccccc}
\hline $\begin{array}{c}\text { 理想气体常数 } \\
R /\left(\mathrm{kg} \cdot \mathrm{m}^{-3}\right)\end{array}$ & $\begin{array}{c}\text { 大气压强 } \\
P / \mathrm{MPa}\end{array}$ & $\begin{array}{c}\text { 气体摩尔质量 } \\
M /\left(\mathrm{g} \cdot \mathrm{mol}^{-1}\right)\end{array}$ & $\begin{array}{c}\text { 腔室压强 } \\
P / \mathrm{MPa}\end{array}$ & $\begin{array}{c}\text { 腔室温度 } \\
T /{ }^{\circ} \mathrm{C}\end{array}$ \\
\hline 8.314 & 0.1 & 28.9 & 0.1 & 14.7 \\
\hline
\end{tabular}

在有限元模拟中，空气弹簧通常被近似为不可 压缩材料, 不可压缩常数 $D$ 由式(18)计算

$$
D=\frac{1-2 \mu}{C_{10}+C_{01}}
$$

由于橡胶是一种超弹性材料, 泊松比约为 0.5 , 因此对本计算, $D$ 设置为 0 。空气弹簧在压缩位移 $20 \mathrm{~mm}$, 充入 $0.2 \mathrm{MPa}$ 气体时的弹射展开过程仿真 结果如图 3 所示。

\section{3 模型验证}

\section{1 空气弹簧弹射试验系统与装置}

为验证仿真模型的正确性, 开展了室温 $14.7{ }^{\circ} \mathrm{C}$ 下的空气弹簧弹射试验, 试验系统组成示意及实物 装置如图 4 所示。系统由空气压缩机、除湿器、储 气缸体、空气弹簧、试验台车、弹射控制器、牵引 钢丝绳、轨道梁、固定刚性墙和固定刚性梁等组成, 在初始状态下空气弹簧一端与储气缸体相连通, 另 一端封闭端面与试验台车相接触。

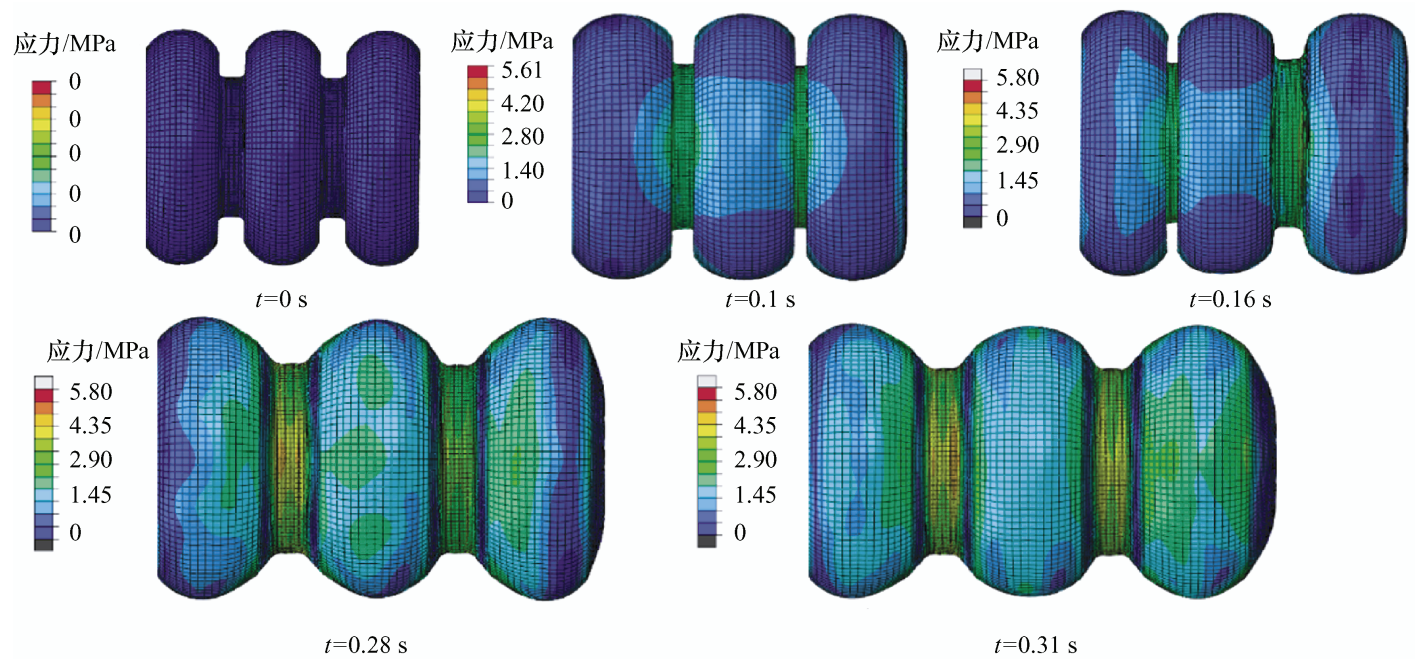

图 3 空气弹簧展开过程 
空气弹簧首先受到弹射控制器约束, 可实现对 空气弹簧的压缩位移量控制, 然后由空气压缩机向 储气缸体充入气体, 达到指定压强后, 通过关闭弹 射控制器实现试验台车约束释放, 在膨胀气体的作 用下, 推动试验台车加速弹射, 最终空气弹簧与移 动体在短时间内脱离接触, 完成弹射。

\section{2 试验测试与仿真对比分析}

图 5 给出了弹射冲击试验中高速摄像机拍摄的 空气弹簧弹射全过程, 包含从受压的初始状态到与 试验台车分离的弹射完成状态, 粗虚线表示空气弹 簧端部运动位置, 细虚线 $a$ 表示空气弹簧压缩后初 始位置。结果表明: 空气弹簧有序展开, 推动试验 台车加速弹射。 $t_{0}$ 时刻到 $t_{5}$ 时刻推动试验台车加速 弹射, $t_{5}$ 时刻到 $t_{6}$ 时刻运动方向发生改变, 说明空 气弹簧已与移动体脱离接触, $t_{6}$ 时刻到 $t_{7}$ 时刻运动 方向再次发生改变, 表明空气弹簧在与台车脱离接 触后发生振荡性伸缩。

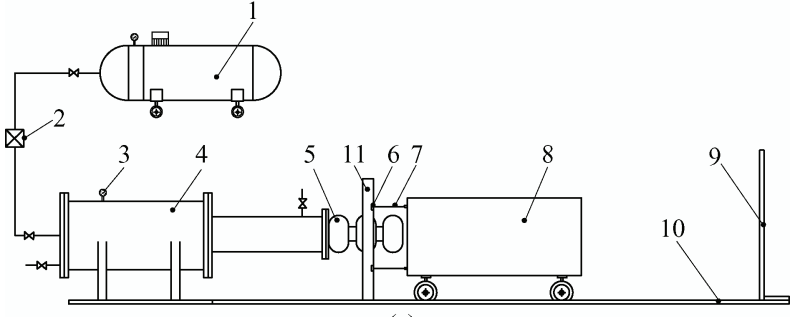

(a)

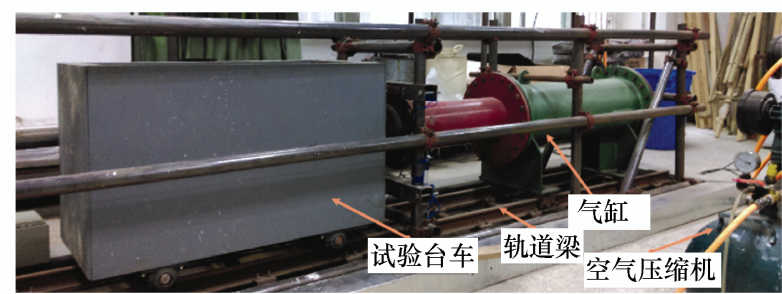

(b)

图 4 空气弹簧弹射试验系统组成示意图

1. 空气压缩机 2. 除湿器 3. 压力表 4. 储气缸体 5 . 空气弹簧 6. 弹射控制器 7. 牵引钢丝绳 8. 试验台车 9. 固定刚性墙 10. 轨道梁 11 . 固定刚性梁

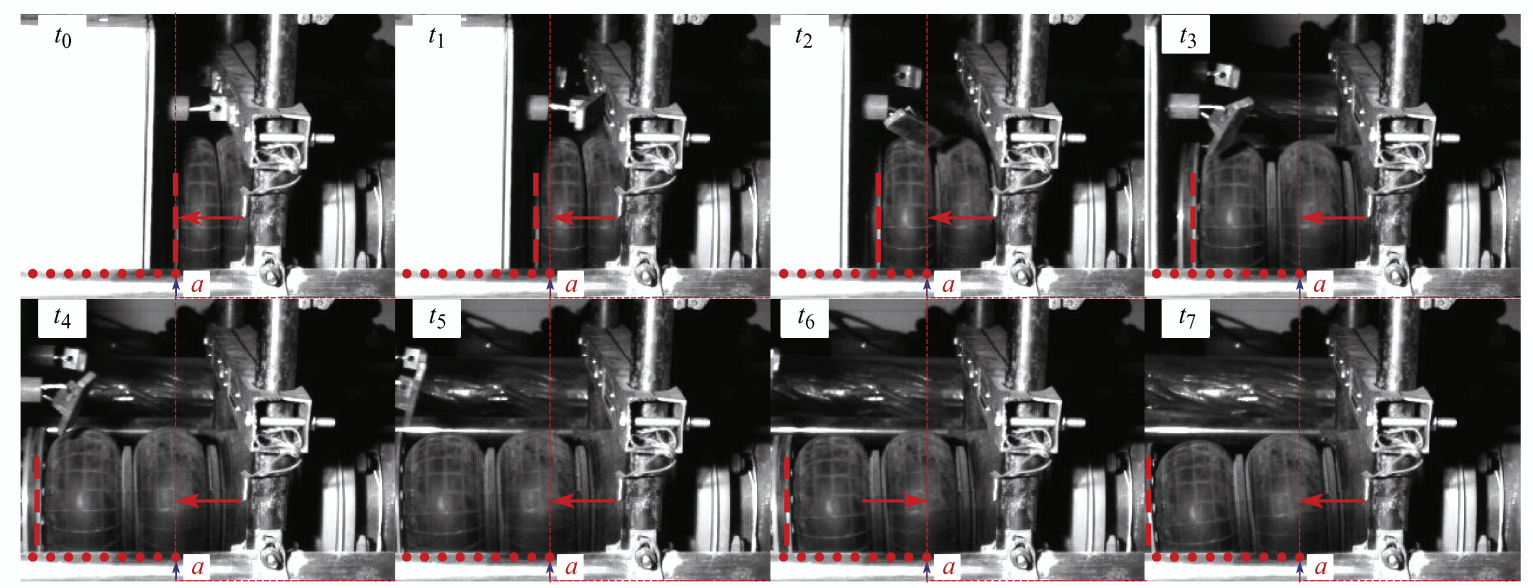

图 5 空气弹簧弹射冲击过程

为验证仿真模型的适用性, 对试验和仿真数据 进行比较。考虑到弹射速度是衡量空气弹簧弹射冲 击性能最重要的指标, 也是研究车辆耐撞性的关键 参数, 因此对比主要围绕弹射速度展开。

由于轨道梁对试验台车有一定约束，使得其他 方向的摆动对弹射速度影响很小，因此在仿真计算 中约束了移动体的其他五个自由度。台车由钢材料 构成, 在弹射过程中几乎没有变形, 在仿真计算中 采用移动体(刚性薄板)代替试验台车，这与实际情 况相符。

通过试验研究不同压缩位移 $(20 \mathrm{~mm} 、 40 \mathrm{~mm}$ 、 $60 \mathrm{~mm}$ 和 $80 \mathrm{~mm}$ )对弹射性能的影响, 空气弹簧弹 射的初始压强均为 $0.2 \mathrm{MPa}$ 。通过速度传感器测得 移动体的弹射速度, 仿真一试验数据对比结果如 图 6 所示。
由图 6 可知, 试验测试结果与仿真计算结果在 变化趋势和数值大小上均十分接近，有效验证了仿 真模型的正确性。

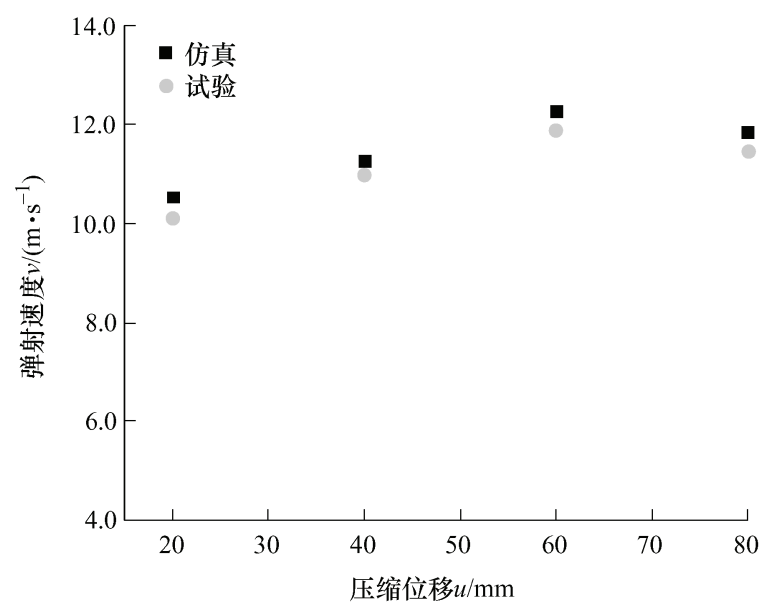

图 6 试验-仿真弹射冲击速度对比 


\section{4 结果与讨论}

\section{1 初始压强对空气弹簧弹射冲击性能影响}

不同初始压强将会对空气弹簧的弹射冲击性能产 生影响。在给定帘线层参数(表 3)和保持空气弹簧初始 压缩位移为 $20 \mathrm{~mm}$ 的情况下, 对空气弹簧分别通入 $0.1 \mathrm{MPa} 、 0.2 \mathrm{MPa} 、 0.3 \mathrm{MPa}$ 和 $0.4 \mathrm{MPa}$ 的气体。在 $0.01 \mathrm{~s}$ 时释放移动体在 $X$ 方向的平动自由度约束, 空 气弹簧推动移动体加速弹射, 从仿真结果得出不同初 始压强下弹射速度随时间变化关系, 如图 7 所示。

由图 7 可知, 随着初始压强增加, 弹射速度随 之增大, 弹射时间变短, 但弹射速度的增加幅度从 $4.24 \mathrm{~m} / \mathrm{s}$ 减小到 $3.21 \mathrm{~m} / \mathrm{s}$, 增加幅度的降低可能由 空气弹簧弹射时间减少造成的。

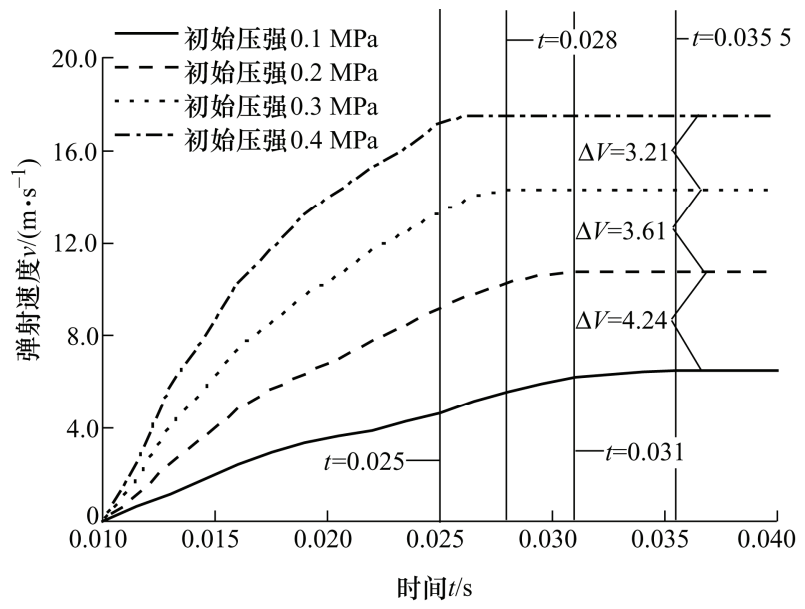

图 7 不同初始压强下弹射速度随时间变化关系

弹射冲击力是衡量空气弹簧弹射冲击性能的重要 指标, 冲击力越大, 弹射速度也越大。从图 8 可看出, 随着初始压强增加, 弹射冲击时间随之变短(冲击力降 为 0 时刻), 这是速度增加幅度降低的主要原因。

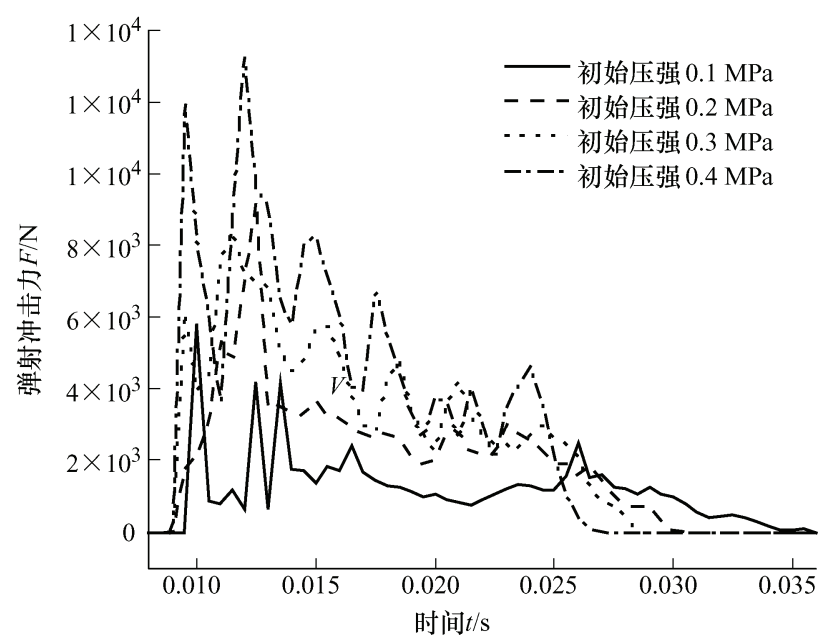

图 8 不同初始压强下弹射冲击力随时间变化关系
不同初始压强下，空气弹簧内部压强随时间变 化如图 9 所示。

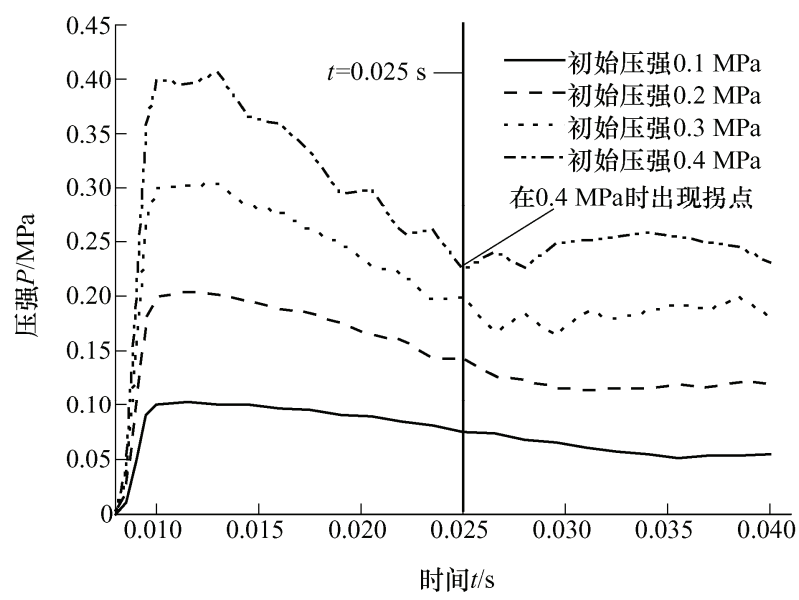

图 9 不同初始压强下空气弹簧内部压强随时间变化关系

由图 9 可知, 空气弹簧内部压强在降低过程中 出现拐点。初始压强越大, 拐点现象越明显。拐点 的出现可能是由空气弹簧与移动体脱离接触后发生 振荡伸缩所引起。

不同初始压强下，空气弹簧弹射过程中内部压 强和体积变化关系如图 10 所示。

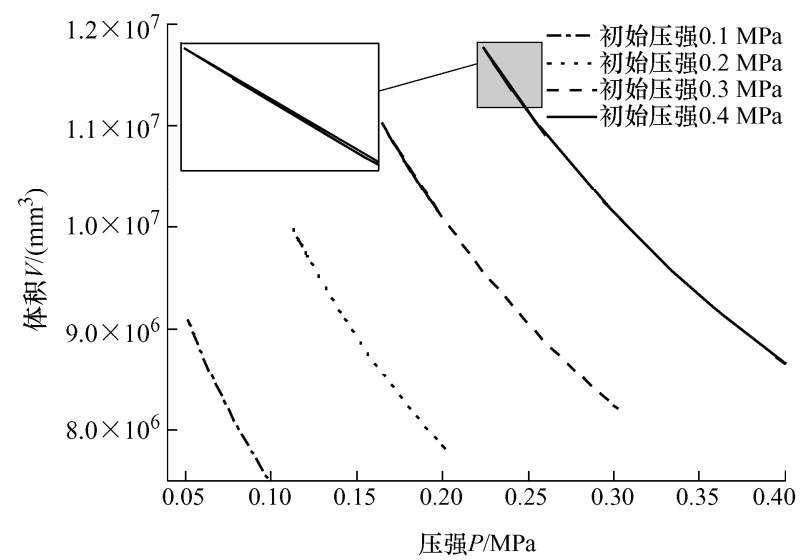

图 10 不同初始压强下空气弹簧内部压强一体积变化关系

空气弹簧内部压强与体积呈近似线性关系，初 始压强越小，线性关系越明显。随着体积增加，压 强也随之降低，在体积达到最大时，振荡随之发生。 从局部放大图可以看出, 腔室体积达到最大后开始 减小，进一步证明了拐点的存在。

为研究空气弹簧腔室温度变化, 在压缩位移 $20 \mathrm{~mm}$, 腔内气体初始温度 $14.7{ }^{\circ} \mathrm{C}$ 参数下, 得出不 同初始压强下空气弹簧内部温度随时间变化如图 11 所示。

由图 11 可见, 内部温度变化有三个不同的阶 段。 $0.008 \mathrm{~s}$ 之前空气弹簧内部温度近似线性增加, 同时温度达到最大值。在 $0.008 \sim 0.010 \mathrm{~s}$, 空气弹 
簧内部温度迅速下降, 初始压强越大, 温度下降速 率就越大。0.01 s 后, 内部温度继续下降, 但下降 速度明显低于第二阶段。空气弹簧与移动体分离后, 随初始压强增加温度波动更加明显, 这是由于空气 弹簧自身振荡性伸缩引起腔内体积不稳定, 进而导 致温度发生波动。

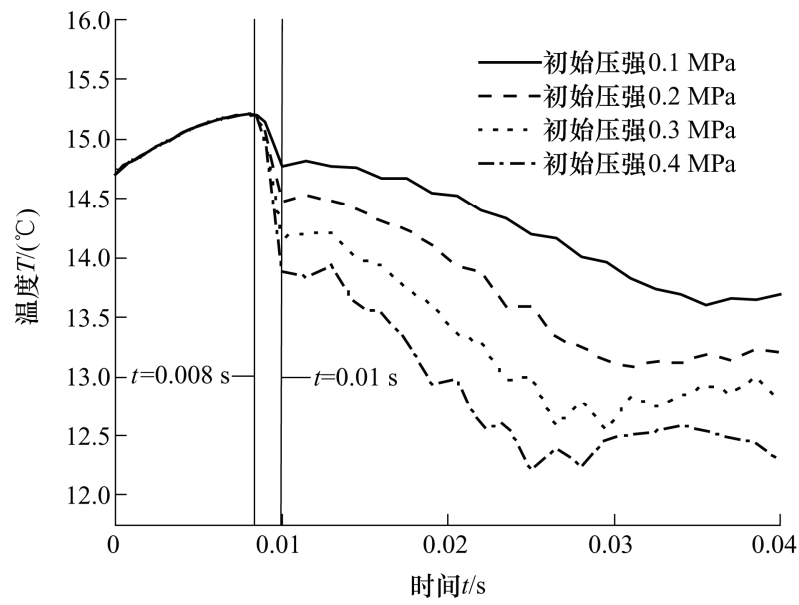

图 11 不同初始压强下腔室温度随时间变化关系

\section{2 压缩位移对弹射冲击性能影响}

为分析压缩位移对弹射冲击性能的影响, 在窝 线层参数均相同的情况下, 分别对初始压强为 $0.1 \mathrm{MPa}$ 和 $0.2 \mathrm{MPa}$ 的空气弹簧施加 $20 \mathrm{~mm}$ 、 $40 \mathrm{~mm} 、 60 \mathrm{~mm}$ 和 $80 \mathrm{~mm}$ 的压缩位移。不同压缩 位移下空气弹簧的弹射速度如图 12 所示。

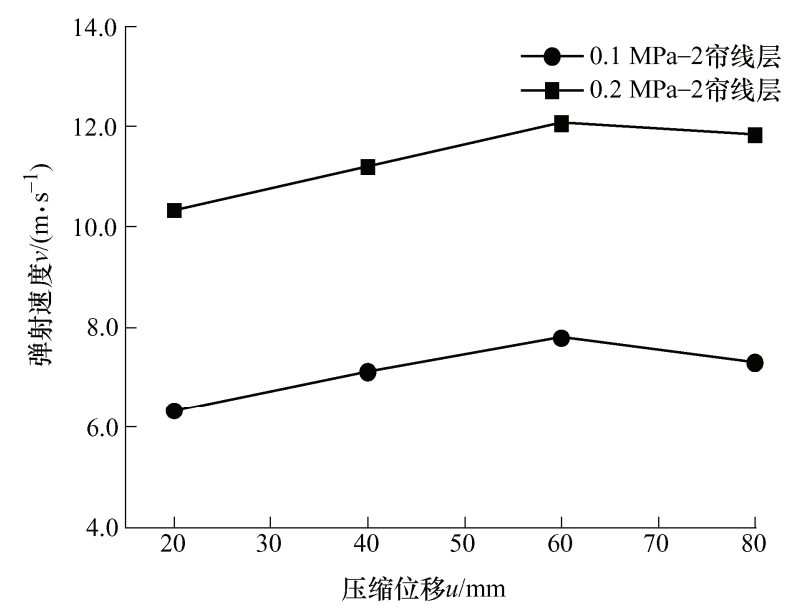

图 12 不同压缩位下空气弹簧对移动体的弹射速度

由图 12 可知, 当压缩位移小于 $60 \mathrm{~mm}$ 时, 压 缩位移与弹射速度近似线性关系，大于 $60 \mathrm{~mm}$ 时, 弹射速度有所降低。无论是空气弹簧内部压强为 $0.1 \mathrm{MPa}$ 还是 $0.2 \mathrm{MPa}$, 弹射速度都有相同的变化 趋势。由此可知, 可以通过增加压缩位移提高弹射 冲击性能, 但压缩位移有一定的限制范围。当超过 此范围时, 弹射冲击性能就会下降。在弹射过程中,
过大的压缩位移可能使空气弹簧不稳定进而导致其 弹射速度降低。

图 13 给出不同压缩位移下空气弹簧在弹射过 程中接触面积与冲击力的变化情况。

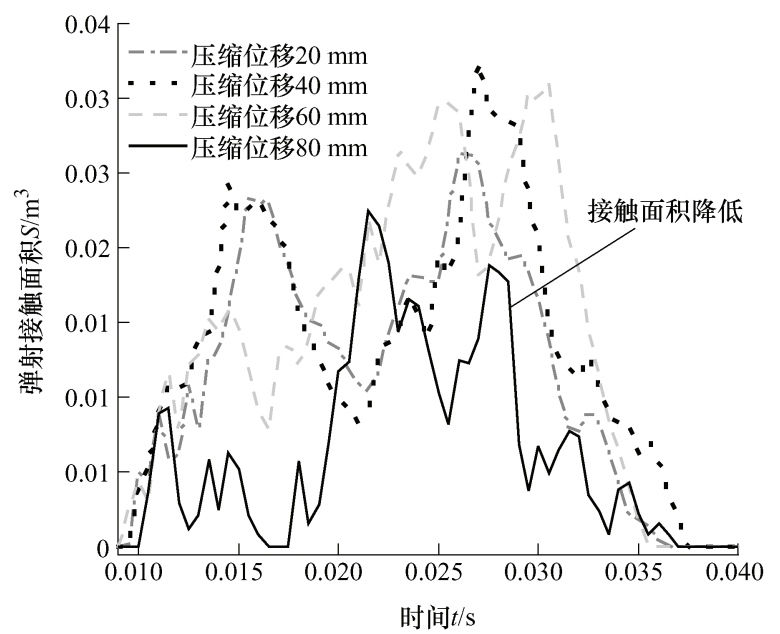

(a)

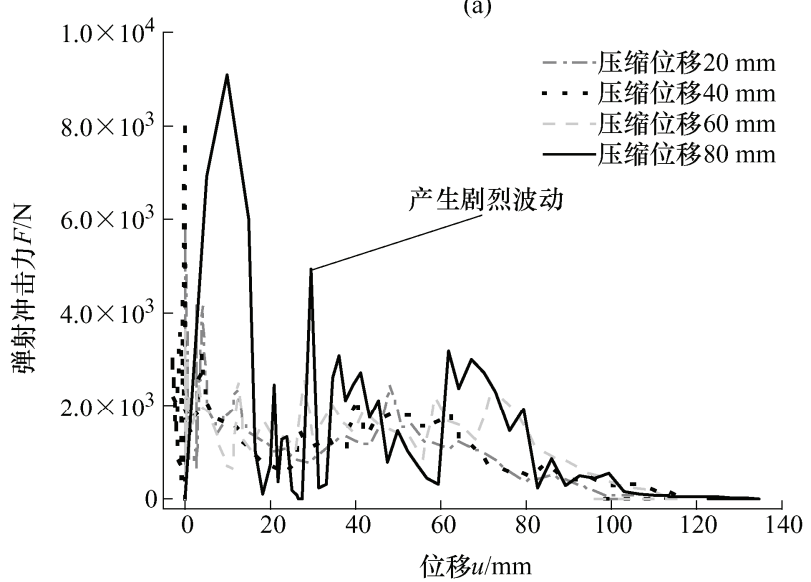

(b)

图 13 不同压缩位移下空气弹簧接触面积与冲击力变化

由图 13 可见，压缩位移为 $80 \mathrm{~mm}$ 时接触面积 明显降低，冲击力波动明显增加。说明过大的压缩 位移导致空气弹簧的稳定性降低。进而验证图 12 中过大的压缩位移导致弹射速度降低的原因。

如图 14 所示, 在空气弹簧上分别建立 3 个径向 节点和 5 个纵向节点, 对位移进行监测, 研究其弹 射过程运动机理。

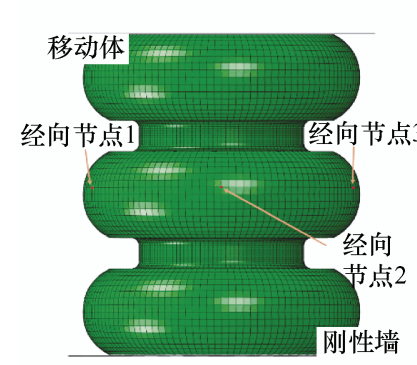

(a)

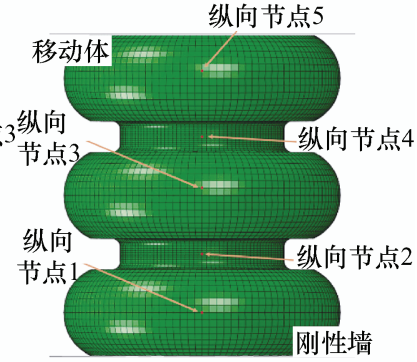

(b)
图 14 径向和纵向节点位置示意图 
在弹射过程中, 空气弹簧径向和纵向节点位移 随时间变化关系如图 15 所示。

由图 15 可知, 径向和纵向节点位移存在显著差 异, 径向节点位移保持一直, 但纵向节点上靠近移动 体的节点位移大于远离移动体的节点位移。随着时间 增加, 径向和纵向节点位移开始波动, 与空气弹簧在 脱离移动体后会发生振荡性伸缩的结论相一致。

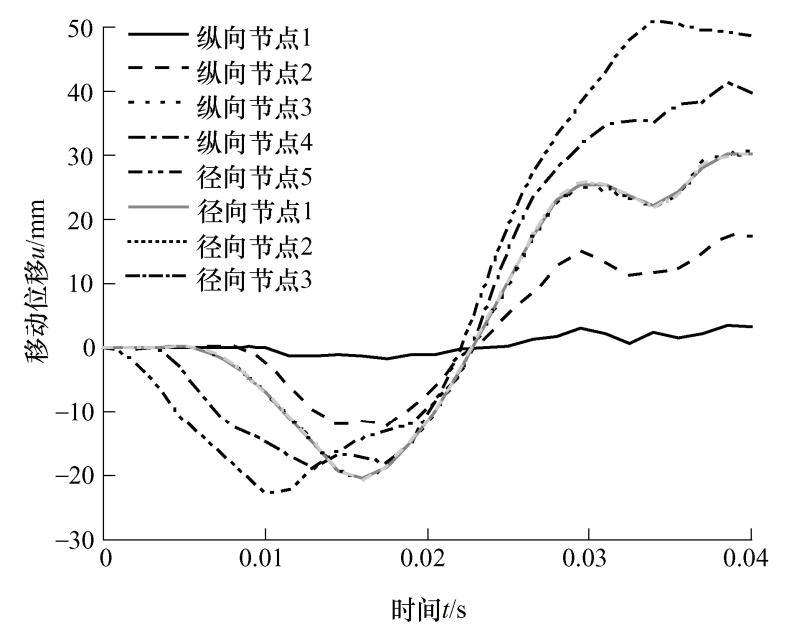

图 15 径向和纵向节点位移随时间变化关系

\section{3 穷线层参数对弹射冲击性能影响}

窝线层是橡胶气囊的承载骨架, 是一种复合 材料, 可以采用橡胶-帘线加强筋模型, 如图 16 所示。加强筋模型可以将橡胶气囊中宋线层表示 在一个 Rebar 单元上, 而不增加自由度。这种方 法在利用三维有限元分析处理几何非线性和物理 非线性问题上非常有效 ${ }^{[29-30]}$ 。本文采用橡胶窝线 加强筋模型, 通过改变帘线直径、间距、层数和 夹角等参数, 分析帘线层参数对空气弹簧弹射冲 击性能的影响。

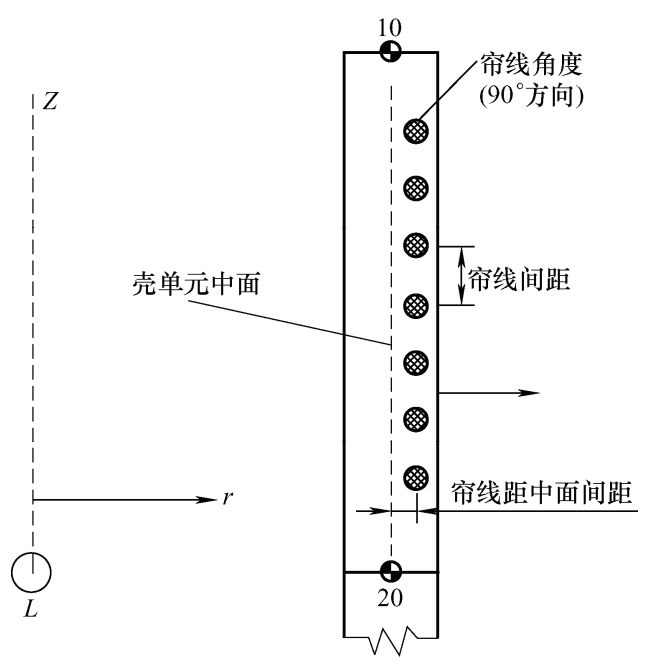

图 16 橡胶-宋线加强筋模型

空气弹簧帘线层初始参数如表 3 所示。帘线层
参数均在相同的初始压强 $0.2 \mathrm{MPa}$ 和压缩位移 $20 \mathrm{~mm}$ 下改变。

表 3 空气弹簧穷线层参数

\begin{tabular}{ccccc}
\hline $\begin{array}{c}\text { 宋线角 } \\
\theta /\left(^{\circ}\right)\end{array}$ & $\begin{array}{c}\text { 层数 } \\
n / \text { 层 }\end{array}$ & $\begin{array}{c}\text { 间距 } \\
d_{1} / \mathrm{mm}\end{array}$ & $\begin{array}{c}\text { 直径 } \\
\varphi / \mathrm{mm}\end{array}$ & $\begin{array}{c}\text { 距中性面 } \\
d_{2} / \mathrm{mm}\end{array}$ \\
\hline 45 & 1 & 1 & 0.2 & 0 \\
\hline
\end{tabular}

不同帘线层参数下空气弹簧的弹射速度与弹射 冲击力分别以图 17、18 为例进行说明。

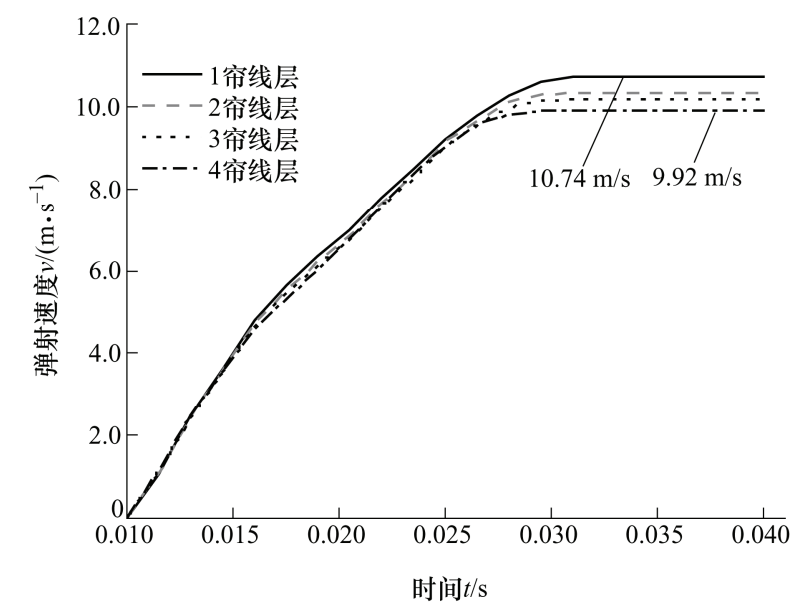

图 17 不同宋线层下空气弹簧对移动体的弹射速度

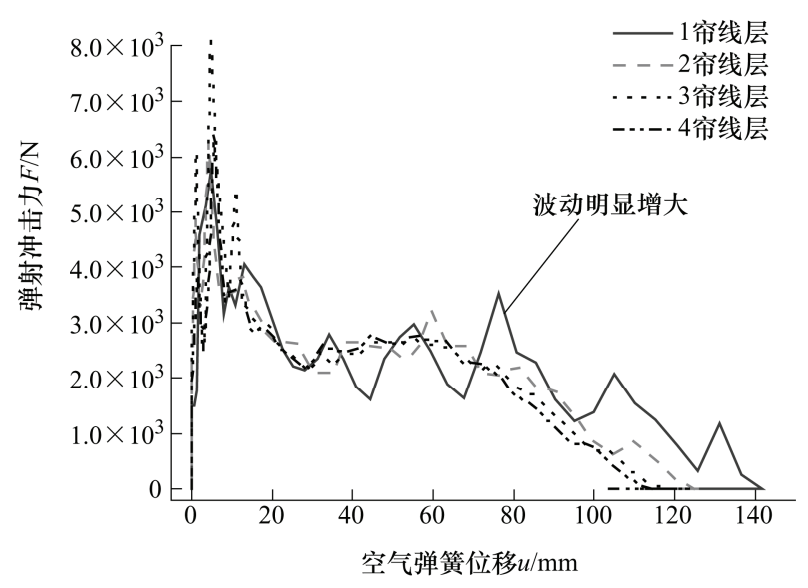

图 18 不同帘线层下的弹射冲击力随位移变化关系

结合图 17、18 可以看出, 弹射速度和弹射冲击 力均随帘线层的增加而降低, 弹射速度从 $10.74 \mathrm{~m} / \mathrm{s}$ 降低到 $9.92 \mathrm{~m} / \mathrm{s}$ 。因此, 减少帘线层可以提高弹射 冲击性能。但 1 宋线层空气弹簧在弹射过程中弹射 冲击力波动明显, 稳定性较差。帘线增加到 2 层及 以上时，空气弹簧的稳定性明显提高。

空气弹簧的弹射速度、冲击力、弹射距离均随 帘线直径的增大而减小，因此，过大的帘线直径不 利于空气弹簧弹射冲击性能的提高。

通过改变帘线角和帘线间距的大小，空气弹簧 弹射速度的提升量均小于 $0.47 \mathrm{~m} / \mathrm{s}$, 同时弹射冲击 力均在同一范围内变化, 由此可知, 帘线角和帘线 
间距的改变对空气弹簧弹射冲击性能影响不大。

综合以上宋线层参数分析可知, 通过改变帘线 层参数获得弹射速度提升量不到 $1 \mathrm{~m} / \mathrm{s}$, 因此宋线 层参数的改变对弹射冲击性能的提升较小, 但通过 增加帘线层数能明显提高空气弹簧的稳定性。

\section{5 结论}

通过弹射冲击试验验证了空气弹簧仿真模型的 有效性, 基于此模型研究初始压强、压缩位移和帘 线层参数对空气弹簧弹射冲击性能的影响。得出如 下结论。

(1) 初始压强和压缩位移对空气弹簧弹射性能 有显著影响, 通过增加初始压强和压缩位移, 可进 一步提高空气弹簧的弹射性能, 但压缩位移不能超 过 $60 \mathrm{~mm}$ 。

(2) 空气弹簧与移动体脱离接触后会出现振荡 性伸缩, 试验和仿真均证实振荡现象的存在, 并通 过仿真计算结果详细说明了振动现象产生的原因。

(3) 帘线层参数对空气弹簧的弹射冲击性能的 影响较小, 但可以通过改变其帘线参数来增强空气 弹簧的抗压强度, 进而通过增加空气弹簧的初始压 强来提高空气弹簧的弹射冲击性能。

\section{参 考 文 献}

[1] 谢俊, 郭晨海, 刘军. 非线性悬架动力学数值模拟和性 能评价[J]. 江苏大学学报, 2004, 25(3): 216-219.

XIE Jun, GUO Chenhai, LIU Jun. Performance evaluation and numerical simulation for non-linear dynamic suspension system of vehicles[J]. Journal of Jiangsu University, 2004, 25(3): 216-219.

[2] 吴兴文, 池茂儒, 朱旻吴, 等. 空气弹簧模型对铁道车 辆动力学性能的影响 [J]. 交通运输工程学报, 2013, 13(2): 54-59.

WU Xingwen, CHI Maoru, ZHU Minhao, et al. Influences of air spring models on dynamic performance of railway vehicle[J]. Journal of Traffic and Transportation Engineering, 2013, 13(2): 54-59.

[3] RYABOY V M. Static and dynamic stability of pneumatic vibration isolators and systems of isolators[J]. Journal of Sound and Vibration, 2014，333(1): 31-51.

[4] 陈燎, 周孔元, 李仲兴. 空气弹簧动态特性拟合及空气 悬架变刚度计算分析 [J]. 机械工程学报, 2010, 46(4): 93-98.

CHEN Liao, ZHOU Kongkang, LI Zhongxing. Dynamic characteristics fitting of air springs and numerical analysis of air suspensions with variant stiffness[J]. Journal of Mechanical Engineering, 2012， 46(4): 93-98.

[5] FACCHINETTI A, MAZZOLA L, ALFI S, et al. Mathematical modeling of the secondary air spring suspension in railway vehicles and its effect on safety and ride comfort[J]. Veh. Syst. Dyn., 2010, 48(1): 429-449.

[6] CHANG F, LU Z H. Dynamic model of an air spring and integration into a vehicle dynamics model[J]. Proceedings of the Institution of Mechanical Engineers. Part D: Journal of Automobile Engineering, 2008, 222(10): 1713-1825.

[7] ZENG X H, ZHANG L, YU Y, et al. The stiffness and damping characteristics of a dual-chamber air spring device applied to motion suppression of marine structures[J]. Applied Sciences, 2016, 6(3): 74.

[8] LI X B, HE Y, LIU W Q, et al. Research on the vertical stiffness of a rolling lobe air spring[J]. Journal of Rail and Transit, 2016, 230(4): 1172-1183.

[9] BHATTACHARYYA S, GHOSH A, BASU B. Nonlinear modeling and validation of air spring effects in a sealed tuned liquid column damper for structural control[J]. Journal of Sound and Vibration, 2017，410：269-286.

[10] 李䒾, 付茂海, 黄运华. 空气弹簧动力学特性参数分析 [J]. 西南交通大学学报, 2003(3): 276-281.

LI Fei, FU Maohai, HUANG Yunhua. Analysis of dynamic characteristic parameter of air spring[J]. Journal of Southwest Jiaotong University，2003(3)：276-281.

[11] 顾太平, 何琳, 赵应龙. 囊式空气弹簧平衡性分析 $[\mathrm{J}]$. 机械工程学报， 2011，47(3): 69-72.

GU Taiping, HE Lin, ZHAO Yinglong. Equilibrium performance analysis for bellows type air spring[J]. Journal of Mechanical Engineering, 2011 , 47(3): 69-72.

[12] ZHU H J, YANG J, ZHANG Y Q, et al. A novel air spring dynamic model with pneumatic thermodynamics, effective friction and viscoelastic damping $[\mathrm{J}]$. Journal of Sound and Vibration, 2017, 408: 87-104.

[13] QUAGLIA G, GUALA A. Evaluation and validation of an air spring analytical model[J]. International Journal of Fluid Power, 2003, 4(2): 43-54.

[14] 徐国敏, 周炜, 何琳, 等. 新型长方体形囊式空气弹簧 耐压强度研究 [J]. 应用力学学报, 2018, 35(2): 436-439, 460.

XU Guomin, ZHOU Wei, HE Lin, et al. Compressive strength of rectangular bellow-type air spring[J]. Chinese Journal of Applied Mechanics, 2018，35(2): 436-439, 460. 
[15] 王千叶. 轨道车辆撞击试验台气动弹射装置的设计研 究[D]. 长沙: 中南大学, 2014.

WANG Qianye. Design research on pneumatic ejection device of rail vehicle crash test equipment[D]. Changsha: Central South University, 2014.

[16] 李建阳, 王红岩, 芮强, 等. 自充气式气蓑缓冲特性试 验研究 [J]. 振动与冲击, 2014, 33(4): 119-123.

LI Jianyang, WANG Hongyan, RUI Qiang, et al. Tests for cushioning characteristics of a self-inflating airbag[J]. Journal of Vibration and Shock, 2014, 33(4): 119-123.

[17] 袁春元, 周孔立, 吴琳琪, 等. 车用空气弹簧有限元分 析方法[J]. 机械工程学报, 2009, 45(6): 262-266.

YUAN Chunyuan, ZHOU Kongkang, WU Linqi, et al. Finite element method to analyze vehicle air spring[J]. Journal of Mechanical Engineering, 2009, 45(6): 262-266.

[18] MELO F, PEREIRA A, MORAIS A. The simulation of an automotive air spring suspension using a pseudodynamic procedure[J]. Applied Sciences, 2018, 8(7): 1049.

[19] 吴善跃, 黄映云, 朱石坚. 空气弹簧冲击载荷特性的试 验研究 $[J]$. 振动与冲击, 2006, 25(2): 113-116, 189. WU Shanyue, HUANG Yingyun, ZHU Shijian. Experimental research on air spring's shock load characteristics[J]. Journal of Vibration and Shock, 2006，25(2): 113-116, 189.

[20] 柳忠彬, 肖守讷, 罗世辉. 列车碰撞试验台气囊弹射加 速仿真研究 [J]. 西南交通大学学报, 2017, 52(1): 127-132.

LIU Zhongbin, XIAO Shoune, LUO Shihui. Simulation of airbag ejection acceleration process on a train collision tested[J]. Journal of Southwest Jiaotong University, 2017, 52(1): 127-132.

[21] LI Y R, XIAO S N, YANG B, et al. Study on the influence factors of impact ejection performance for flexible airbag[J]. Advances in Mechanical Engineering, 2018, 10(10): 1-11.

[22] ALONSO A, GIMENEZ J G, NIETO J, et al. Air suspension characterization and effectiveness of a variable area orifice[J]. Veh. Syst. Dyn., 2010, 48(S1): 271-286.

[23] GODA K, NISHIGAITO T, HIRAISHI M, et al. A curving simulation for a monorail $\operatorname{car}[\mathrm{C}] / /$ Proceedings of the ASME/IEEE Joint Railroad Conference, Newark, NJ, USA, 6-6 April 2000, IEEE Xplore, 06 August 2002, 171-177.

[24] 方康寿. 无人机回收气囊减震性能的有限元研究 [D]. 杭州: 浙江大学, 2008.

FANG Kangshou. The FEA research on a seismic performance of recovery airbag on UAV[D]. Hangzhou: Zhejiang University, 2008.

[25] DIJK R, KEULEN F, STERK J C. Simulation of closed thin-walled structures partially filled with fluid[J]. International Journal of Solids and Structures, 2000, 37(42): $6063-6083$.

[26] BERRY D. Formulation and experimental verification of pneumatic and hydraulic finite elements[D]. West Lafayette: Purdue University, USA, 1994.

[27] HIREMATH S S, SINGAPERUMAL M. Investigations on actuator dynamics through theoretical and finite element approach[J]. Mathematical Problems in Engineering, 2010, 2010: 1-22.

[28] MOONY M. A theory of large elastic deformation[J]. J. Appl. Phys., 2004, 11(9): 582-592.

[29] SPRENGER W, WAGNER W. On the formulation of geometrically nonlinear 3D-Rebar-elements using the enhanced assumed strain method[J]. Engineering Structures, 1999, 21(3): 209-218.

[30] MESCHKE G, HELNWEIN P. Large-strain 3D analysis of fibre-reinforced composites using rebar elements: Hyperelastic formulations for cords[J]. Computational Mechanics, 1994, 13(4): 241-254.

作者简介: 李玉如, 男, 1987 年出生, 博士研究生。主要研究方向为车 辆被动安全。

E-mail: liyuru@my.swjtu.edu.cn

杨冰(通信作者), 男, 1979 年出生, 博士, 研究员, 博士研究生导师。 主要研究方向为结构强度理论与可靠性、材料疲劳与断裂。

E-mail: yb@swjtu. edu.cn 